\title{
Psychometric properties of the interRAI subjective quality of Life Instrument for mental health
}

\author{
Tess E. Naus ${ }^{1}$, John P. Hirdes ${ }^{2^{*}}$ \\ ${ }^{1}$ Department of Mood Disorders, PsyQ, Parnassia Groep, The Hague, The Netherlands \\ ${ }^{2}$ School of Public Health and Health Systems, University of Waterloo, Waterloo, Canada; \\ *Corresponding Author: hirdes@uwaterloo.ca
}

Received 9 January 2013; revised 15 February 2013; accepted 23 February 2013

\begin{abstract}
A new Subjective Quality of Life (SQoL) Instrument for inpatient and community mental health settings was developed by the interRAI research collaborative to support evaluation of quality in mental health settings from the person's perspective. Ratings of SQoL provide important information about the quality of service and patient experience with the care they receive. This information can help staff to improve approaches to each person's plan of care in a manner that is meaningful to the individual. This study examined the reliability of the SQoL-MH. 83 inpatients from several clinical departments in a mental health center in South Western Ontario, Canada were randomly assigned to either be interviewed or complete the assessment on his or her own. Reliability was tested using Cronbach's Alpha. A preliminary factor analysis points to four SQoLMH subscales with very good internal consistency, ranging from 0.83 to 0.90 . Once finalized, the Subjective Quality of Life instrument will be integral to the interRAI suite of instruments used to assess persons with mental health needs. A reliable and valid SQoL-MH instrument will allow mental health service providers to shape or modify care environments in order to enhance quality of life. In addition, the SQoL-MH instrument could also benefit advocacy groups who use reports on quality of life to influence social policy development and funding decisions.
\end{abstract}

Keywords: interRAI; Quality of Life; Quality of Care; Recovery

\section{INTRODUCTION}

Severe mental illness may have a major, broad impact on a person's life [1]. It is therefore important to be able to assess accurately the person's complex needs and to meet them as effectively as possible. The person's subjective appraisal of quality of life (QoL) is an important consideration in any evaluation of the extent to which this has been achieved [2]. However, a clear universal definition of this concept does not exist for the general population, and even less consensus is evident with respect to persons with mental health needs.

\subsection{Different Approaches to QoL}

There are different approaches to quality of life measurement. One of the earliest approaches focused mostly on consideration of objective life circumstances (for example, income, employment and housing conditions). More sophisticated indicators of objective quality of life also included information on a person's social network, and opportunities for leisure and recreation [3]. Healthrelated Quality of Life (HRQoL) is one of the more conventional ways to measure quality of life and has been defined as "the value assigned to duration of life as modified by the impairments, functional states, perceptions, and social opportunities that are influenced by disease, injury, treatment, or policy" [4]. Health and economic approaches to quality of life often focus on QALYS (Quality-Adjusted Life Years) and DALYS (DisabilityAdjusted Life Years), which are developed to measure health outcomes in a standardized way that takes into account both the quantity and quality of life generated by healthcare innovations [3]. These measures are often used in cost-utility analyses, pharmacoeconomics, and allocation of healthcare resources. Another approach to quality of life-one that is often used in social sciences, gerontology and social psychology-focuses on subjective global well-being, which is sometimes equated with psychosocial well-being, happiness and life satisfaction [5]. There is a large literature on this subject, which is mostly atheoretical and shows little agreement on methods and concepts. Hence, a clear universal definition of QoL is still lacking. 


\section{2. interRAI Quality of Life Instruments}

A new Subjective Quality of Life Instrument (SQoL) for inpatient and community mental health settings has been developed by the interRAI research group (www.interrai.org) as a complementary tool to existing assessor rated clinical assessments. interRAI is a notfor-profit collaborative network of researchers in over 30 countries committed to improving health care for vulnerable populations, including those affected by mental illness. The goal of interRAI is to promote evidenceinformed clinical practice and policy decisions through the collection and interpretation of high quality data about the characteristics and outcomes of persons served across a variety of health and social services settings. As part of their international effort to expand an integrated suite of assessment instruments that is now used extensively in Canada, interRAI has developed SQoL instruments for mental health, home and community care, and long-term care home settings [6-8]. Large-scale pilots of the home care and nursing home instruments have been conducted in several countries, including Canada. For example, a recent national survey of 48 nursing homes with a sample of over 900 residents who responded to the interRAI QoL survey for that sector [9]. The interRAI family of assessment instruments treat QoL as a multidimensional concept. As discussed above, there are two broad perspectives on quality of life: the objective one, which stresses externally-knowable aspects (for example, employment, housing status, extreme poverty or abuse) and the subjective dimension, which focuses on the idiosyncratic part of quality of life (self-ratings, an individual's unique perspective). These two perspectives do not necessarily always coincide but neither perspective can be ignored. The assessor rated interRAI instruments consider the objective dimensions of QoL, where- as the SQoL survey considers only the person's perspective in rating different dimensions of the person's experience. These instruments do not only consider HR QoL, but rather they address a much broader range of the person's experiences. The interRAI-SQoL-MH focuses on the subjective part of quality of life and aims to give persons who use mental health services the opportunity to speak for themselves and express their own views about their lives. Although quality of life is a characteristic of the person rather than of the interventions, treatments or services he or she receives, changes in quality of life may be relevant outcome indicators of the quality of care. For example, Ruggeri and colleagues [10] found that patients' experience of community mental health services was positively associated with subjective quality of life. One would also expect that relationships with staff and access to necessary services would have a substantial impact on the quality of life of mental health service users. Therefore, the interRAI-SQoL-MH instrument also includes items on staff relationships (Table 1). The new SQoL-MH instrument is concise: it consists of ten domains with less than 50 items in total and differs therefore from other instruments that have many more items [11-14]. Items of the interRAI-SQoL-MH instrument were based on feedback from clinical staff of mental health care settings in several countries and on a review of the literature. The literature on recovery from mental illness was an important basis for development of the content of the interRAI SQoL-MH instrument. There are many definitions of the term "recovery" in the literature. For instance, recovery has been defined as "persons with severe mental illness living a satisfying life within the constraints of one's mental illness" [15, p. 1035]. Andresen, Oades and Caputi [16] propose a consumer-oriented definition of recovery: "the establishment of a ful

Table 1. Key domains of SQoL-MH.

\begin{tabular}{|c|c|}
\hline Domains & Sample item \\
\hline Identification Information (5 items) & Birthdate, gender, length of stay \\
\hline Personal Outlook (5 items) & I am hopeful about my future \\
\hline Autonomy and Self-determination (5 items) & I am in control of my thoughts and feelings \\
\hline Meaningful Activities (4 items) & I participate in meaningful activities \\
\hline Friends and Family (5 items) & My relationships with family and friends are good \\
\hline Community (4 items) & I participate in community activities \\
\hline Staff relationships (4 items) & Staff support my recovery \\
\hline Privacy (4 items) & My privacy is respected by staff \\
\hline Empowerment and Support (4 items) & I get support for the decisions I make about my life \\
\hline Access to Services (4 items) & If I need help right away, I can get it \\
\hline
\end{tabular}


filling, meaningful life and a positive sense of identity founded on hopefulness and self-determination”. They identified four key processes of recovery: 1) finding and maintaining hope; 2) the reestablishment of a positive identity; 3) finding meaning in life; and 4) taking responsibility for one's life (p. 558). The concept of quality of life includes many of the same concepts referred to in the recovery literature [17] and several studies have found recovery to be associated with quality of life [18, 19]. Therefore, the interRAI-SQoL instrument includes items about autonomy and self-determination, meaningful activities, and empowerment and support (Table 1).

\subsection{Pilot Study}

Ratings of quality of life of persons receiving care in mental health inpatient care settings provide important information about the quality of service and satisfaction with the care they are receiving. Although some dimensions of quality of life are externally observable by raters other than the person, as noted before quality of life also includes subjective aspects that are unique to each individual. Therefore, self-reported ratings are important measures to include in any effort to evaluate quality of life. These measures can help staff to improve approaches to each person's plan of care in a manner that is meaningful to the individual. In addition, quality of life information may be aggregated to provide information that may be used for benchmarking purposes at the organizational level as part of quality improvement initiatives.

From a clinical point of view, factors that are shown to be associated with poorer quality of life may

be appropriate targets for change strategies aimed at improving the lives of persons receiving mental health services. On the other hand, some mental health characteristics might also be appropriate to use as risk adjusters when benchmarking the performance of diverse mental health organizations. For example, in organizations providing services to a population with more severe cognitive impairment or psychosis, quality of life ratings may be different from those in other organizations at least in part as a function of patient characteristics rather than service quality alone.

The purposes of this pilot study were to examine the reliability of the interRAI SQoL-MH instrument and to compare the data quality and response patterns for the quality of life ratings obtained via direct in person interviews with self-administered responses.

\section{METHODS}

\subsection{Participants}

Participants are 83 inpatients from a mental health center in South Western Ontario, Canada $(\mathrm{N}=83,36$ males, 47 females, aged 19 - 71 years old $(M=43.67$,
$S D=11.75)$ ), from several departments (comprehensive psychiatric care, eating disorders, alcohol and drug program, integrated mood and anxiety program, post-traumatic stress recovery program). Persons with a substitute decision maker, persons with vision and/or hearing impairments, persons with cognitive impairment (CPS score of 4 or more, RAI-MH [20]) and persons who hospital staff have identified as unable to provide informed consent for participation in the study were excluded from this study.

\subsection{Materials}

The interRAI Subjective Quality of Life instrument for Mental Health (SQoL-MH) contains less than 50 items and asks respondents to rate the frequency of occurrence of different aspects of their quality of life (Table 1). The only responses to be recorded are those given by the person. Interviewer judgment is not used to interpret the person's experience.

\subsection{Procedure}

Participants who had indicated their willingness to participate in the study received an information letter explaining the study purpose, procedure, and risks and benefits of participating. Once informed consent had been obtained, participants were randomly assigned to either be interviewed or to be asked to complete the form on his or her own. All interviewers were trained in the study protocol and the use of the SQoL-MH. Both the interview and the self-administration took place in a private location that allowed the participants to have an appropriate level of privacy to complete the study. The centre's staff had no access to the person's survey data in order to ensure that ratings about the staff would remain confidential.

\subsection{Analysis}

Examination ofinternal consistency, descriptive analyses and factor analyses were carried out using Statistical Analysis Software (SAS ${ }^{\circledR}$ 9.1).

\subsection{Ethics}

Full ethical clearance was obtained for this study from both the Office of Research Ethics of the University of Waterloo and the centre's research ethics board (ORE \#16707, 10/14/2010).

\section{RESULTS}

\subsection{Reliability}

A Cronbach's alpha value of 0.70 or higher was considered to be evidence of good reliability [21]. As shown 
in Table 2, the Cronbach alpha values ranged from 0.62 (meaningful activities) to 0.87 (interpersonal relationships) for the ten domains of the instrument. This indicates that for all but the one domain (Meaningful Activities), internal consistency was considered to be good.

A preliminary factor analysis using a principal component extraction method and a varimax rotation of the 46 SQoL-MH items points to four subscales. Factor 1, labeled staff relations, (eigenvalue $=14.11$ ) accounted for $31 \%$ of the variance and had nine items (e.g., "Staff listen to what I have to say”, $\alpha=0.88$ ). Factor 2, labeled personal outlook and community engagement, (eigenvalue $=4.66$ ) accounted $10 \%$ of the variance and consisted of eight items (e.g., "On the whole, my life is good”, $\alpha=0.90)$. Factor 3 , labeled interpersonal relationships, (eigenvalue $=2.70$ ) accounted for $6 \%$ of the variance and had six items (e.g., "I am an important part of other people's lives”, $\alpha=0.86$ ). Finally, factor 4, labeled meaningful activities, (eigenvalue $=2.38$ ) accounted for $5 \%$ of the variance and had five items (e.g., "I am motivated to participate in everyday activities", $\alpha$ $=0.83)$.

Descriptive analyses of the preliminary subscales indicate that participants rate their relations with staff higher than they rate their own lives, as shown in Figure 1. The participants seem to be particularly satisfied with the way they are treated by the centre's staff. Lower scale scores on the personal life and community engagement scale suggest that participants are less hopeful about their selves, their lives, their future and their community engagement.

\subsection{Administration Method}

Independent sample T-tests were conducted to compare administration types. There was only a marginally significant difference in scores on the meaningful active-

Table 2. Internal consistency of SQoL-MH domains $(\mathrm{n}=83)$.

\begin{tabular}{lc}
\hline Domains & Cronbach Alpha \\
\hline Personal Outlook & 0.88 \\
Autonomy and self-determination & 0.72 \\
Meaningful activities & 0.62 \\
Relationships with friends and family & 0.87 \\
Community engagement & 0.81 \\
Staff relations & 0.84 \\
Privacy & 0.85 \\
Empowerment and support & 0.83 \\
Discrimination and life circumstances & 0.71 \\
Access to services & 0.79 \\
\hline
\end{tabular}

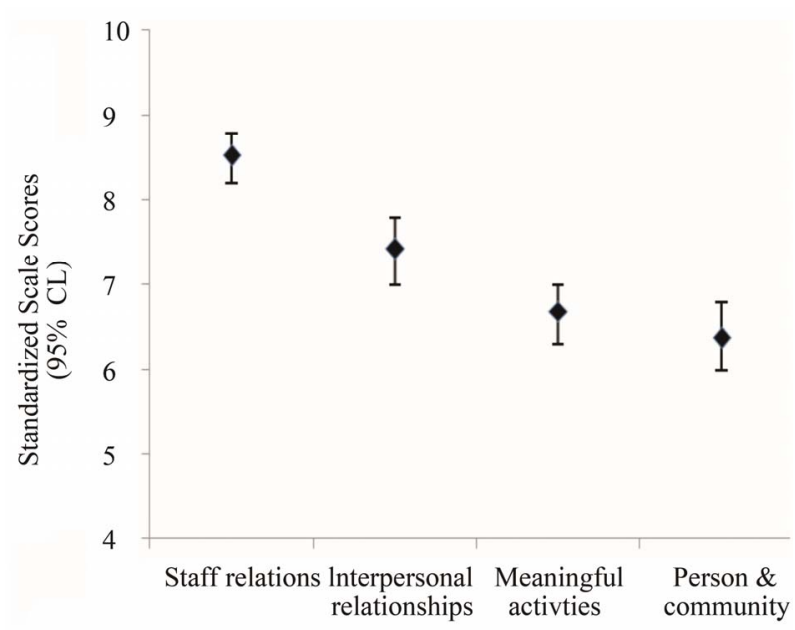

Figure 1. Standardized scale scores, $n=83$ participants.

ties scale $(\mathrm{t}(81)=-1.96, p=0.054)$ with participants who were being interviewed having slightly higher scores $(M=7.13, S D=1.45)$ than those who filled out the questionnaire on their own $(M=6.43, S D=1.63)$.

\section{DISCUSSION}

This pilot study showed that the interRAI-SQoL instrument has good reliability with a diverse inpatient population. In addition, the instrument yielded information that is highly relevant from the perspective of recovery from mental illness, including four subscales that provide summary measures of different aspects of quality of life.

Participants rated their relations with staff more positively than their own lives and futures. Perhaps this was to be expected, given that admission to a psychiatric facility means that one's life may be put on hold and one depends on staff for support. However, it might also be argued that it is important that persons admitted with mental health problems are supported and empowered to finding and maintaining hope and meaning in their lives. Thus, one would have liked to see higher scores on the subscales measuring these qualities.

Comparing the methods of administration showed that participants who were being interviewed were slightly more positive about their meaningful activities then those who filled out the questionnaire on their own. This is consistent with the possibility of a social desirability bias where respondents may be more willing to be frank about their situation when responding with greater anonymity [22].

\subsection{Limitations of This Study}

This pilot study suffers from several limitations. The sample size was sometimes too small to be able to do subgroup analyses. In addition, there was not sufficient 
variability in measures of symptoms related to cognition and psychosis, so it is not possible to determine whether this new instrument is also suitable for those with severe mental illness and/or cognitive problems. The findings of this study may therefore not be generalizable to all per- sons with psychotic disorders.

Since QoL measures are often used as outcome indicators of quality of care and treatment efficacy, these measures should be sensitive to changes in the clinical and social conditions of the person that are targeted by mental health services and interventions. Hansson [22] posed the question: "Is [subjective quality of life] a reflection of internal and external states amenable to the kind of changes that are related to interventions? Or does it reflect naturalistic changes in a person's life situation?” (p. 48). It is also not clear to what extent ratings of SQoL reflect fixed personality traits. It could indeed be expected that a person's underlying personality also determines his or her evaluation of QoL [23-25]

\subsection{Conclusions}

The purposes of this study were to examine the reliability of the new interRAI SQoL-MH instrument in a mental health setting and to compare data quality and response patterns for the SQoL ratings obtained via direct in person interview and those obtained via self-administration. The reliability of the new instrument was quite acceptable in that the alpha values of its ten domains were moderate to high. Preliminary factor analysis points to four subscales with good internal consistency. It should be pointed out that these analyses are preliminary and provisional and more research is needed. In future research the interRAI-SQoL-MH instrument will be tested with more respondents, in more diverse settings and in different countries. A reliable and valid SQoL instrument will allow mental health service providers to shape or modify care environments in order to enhance QoL. In addition, the SQoL-MH instrument could also benefit advocacy groups who use reports on quality of life to influence social policy development and funding decisions.

\section{ACKNOWLEDGEMENTS}

The authors would like to thank Christopher Perlman, Shannon Matkovich, Leslie Eckel, Julie Koreck, Steve De Thomasis, Lizzy Kaempffer, and Sabina Staempfli who all supported this project.

\section{REFERENCES}

[1] Evans, S., Banerjee, S., Leese, M. and Huxley, P. (2007) The impact of mental illness on quality of life: A comparison of severe mental illness, common mental disorder and healthy population samples. Quality of Life Research, 16,17-29. doi:10.1007/s11136-006-9002-6
[2] Lehman, A.F. (1996) Measures of quality of life among persons with severe and persistent mental disorders. Social Psychiatry and Psychiatric Epidemiology, 31, 78-88. doi:10.1007/BF00801903

[3] Holloway, F. and Carson, J. (2002) Quality of life in severe mental illness. International Review of Psychiatry, 14,176-184. doi:10.1080/09540260220145000

[4] Patrick, D.L. and Erickson, P. (1993) Health status and health policy: Quality of life in health care evaluation and resource allocation. Oxford University Press, New York.

[5] Baker, F. and Intagliata, J. (1982) Quality of life in the evaluation of community support systems. Evaluation and Programme Planning, 5, 69-79. doi:10.1016/0149-7189(82)90059-3

[6] Martin, L. and Hirdes, J.P. (2009) Mental health needs and service use in Ontario. Healthcare Management Forum, 22, 40-46. doi:10.1016/S0840-4704(10)60291-8

[7] Hirdes, J.P., Ljunggren, G., Morris, J.N., Frijters, D.H., Finne Soveri, H., Gray L. and Gilgen, R. (2008) Reliability of the interRAI suite of assessment instruments: A 12-country study of an integrated health information system. BMC Health Services Research, 8, 277-288. doi:10.1186/1472-6963-8-277

[8] Hirdes, J.P. (2006) Addressing the health needs of frail elderly people: Ontario's experience with an integrated health information system. Age and Ageing, 35, 329-331. doi:10.1093/ageing/afl036

[9] Kehyayan, V. (2011) Relationships between quality of life and selected resident and facility characteristics in long term care facilities in Canada. Ph.D. Thesis, University of Waterloo, Waterloo.

[10] Ruggeri, M., Gater, R., Bisoffi, G., Barbui, C. and Tansella, M. (2002) Determinants of subjective quality of life in patients attending community-based mental health services. The South-Verona Outcome Project 5. Acta Psychiatrica Scandivica, 105, 131-140. doi:10.1034/j.1600-0447.2002.01293.x

[11] Bigelow, B.A., McFarland, B.H. and Olson, M.M. (1991) Quality of life of community mental health program clients: Validating a measure. Community Mental Health Journal, 27, 43-55. doi:10.1007/BF00752714

[12] Lehman, A.F. (1983) The well-being of chronic mental patients: assessing their quality of life. Archives of General Psychiatry, 40, 369-373. doi:10.1001/archpsyc.1983.01790040023003

[13] Oliver, J.P.J., Huxley, P.J. and Priebe, S. (1997) Measuring the quality of life of severely mentally ill people using the Lancashire quality of life profile. Social Psychiatry and Psychiatric Epidemiology, 32, 76-83. doi:10.1007/BF00788924

[14] Power, M., Kuyken, W., Orley, J., Herrman, H., Schofield, H., Murphy, B. and Sartorius, N. (1998) The World Health Organization Quality of Life assessment (WHOQOL): Development and general psychometric properties. Social Science \& Medicine, 46, 1569-1585. doi:10.1016/S0277-9536(98)00009-4

[15] Corrigan, P.W., Salzer, M., Ralph, R.O., Sangster, Y. and Keck, L. (2004) Examining the factor structure of the re- 
covery assessment scale. Schizophrenia Bulletin, 30, 1035-1041. doi:10.1093/oxfordjournals.schbul.a007118

[16] Andresen, R., Oades, L. and Caputi, P. (2003) The experience of recovery from schizophrenia: Towards an empirically validated stage model. Australian and New Zealand Journal of Psychiatry, 37, 586-594. doi:10.1046/j.1440-1614.2003.01234.X

[17] Lieberman, J.A., Drake, R.E., Sederer, L.I., Belger, A., Keefe, R., Perkins, D. and Stroup, S. (2006) Science and recovery in schizophrenia. Psychiatric Services, 59, 487496. doi:10.1176/appi.ps.59.5.487

[18] Corrigan, P.W., Giffort, D., Rashid, F., Leary, M. and Okeke, I. (1999) Recovery as a psychological construct. Community Mental Health Journal, 35, 231-239. doi:10.1023/A:1018741302682

[19] Chiu, Y.L., Ho, W.N., Lo, T.L. and Yiu, G.C. (2010) Operationalization of the SAMHSA model of recovery: A quality of life perspective. Quality of Life Research, 19, 1-13. doi:10.1007/s11136-009-9555-2

[20] Hirdes, J.P., Marhaba, M., Smith, T.F., Clyburn, L., Mitchell, L., Lemick, R.A. and Yamauchi, K. (2001) Development of the Resident Assessment Instrument-Mental
Health (RAI-MH). Hospital Quarterly, 4, 44-51.

[21] Streiner, D.L. and Norman, G.R. (2003) Health measurement scales: A practical guide to their development and use. Oxford University Press, Oxford,

[22] Kaiser, W. and Priebe, S. (1999) The impact of the interviewer-interviewee relationship on subjective quality of life ratings in schizophrenia patients. International Journal of Social Psychiatry, 45, 292-301. doi:10.1177/002076409904500408

[23] Hansson, L. (2006) Determinants of quality of life in people with severe mental illness. Acta Psychiatrica Scandinavica, 113, 46-50. doi:10.1111/j.1600-0447.2005.00717.x

[24] Eklund, M., Bäckström, M. and Hansson, L. (2003) Personality and selfvariables: Important determinants of subjective quality of life in schizophrenia outpatients. Acta Psychiatrica Scandinavica, 108, 134-143. doi:10.1034/j.1600-0447.2003.00103.x

[25] Zissi, A., Barry, M.M. and Cochrane, R. (1998) A meditational model of quality of life for individuals with severe mental health problems. Psychological Medicine, 28, 1221-1230. doi:10.1017/S0033291798007338 